\title{
CARACTERIZAÇÃO HIDROGEOQUÍMICA DE ÁREA IMPACTADA POR DEPÓSITO DE RESÍDUOS DESATIVADO EM ZONA DE RECARGA DO AQUÍFERO GUARANI
}

\author{
HYDROGEOCHEMICAL CHARACTERIZATION OF AREA IMPACTED BY A DISABLED WASTE \\ DEPOSIT IN A GUARANI AQUIFER RECHARGE ZONE
}

\author{
Alice Kimie Martins MORITA, Edson Cezar WENDLAND \\ Universidade de São Paulo, Escola de Engenharia de São Carlos. Avenida Trabalhador São Carlense, 400 - Parque Arnold Schimidt, \\ São Carlos - SP. E-mail: akmmorita@gmail.com, ew@sc.usp.br

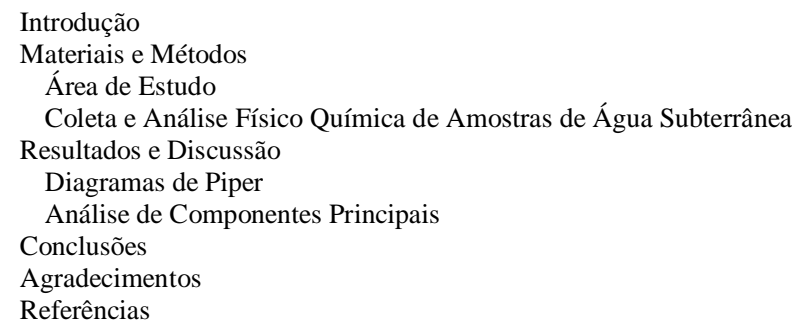

\begin{abstract}
RESUMO - A deposição de resíduos sólidos sem o adequado planejamento pode impactar o entorno por décadas ou séculos após o término das atividades. O impacto das contaminantes lixiviados no meio depende das características da formação geológica, bem como da ocorrência de processos de transporte e atenuação. Este estudo visou a estudar os contaminantes encontrados no entorno de um depósito de resíduos desativado localizado em área de recarga do Aquífero Guarani, bem como suas variações sazonais. Foram realizadas coletas de amostras de água subterrânea em 14 poços de monitoramento em julho e dezembro de 2017, e foram analisados diferentes parâmetros de interesse, como $\mathrm{pH}$, condutividade, alcalinidade, DQO, cloretos, nitrato, nitrogênio amoniacal, ferro, manganês, cálcio e metais potencialmente tóxicos. Verificaram-se altas concentrações de contaminantes no interior do depósito de resíduos, mas considerável redução à jusante. Ainda assim, observou-se nítida influência do depósito em seu entorno, com possível ocorrência de reações redox e com variações sazonais da qualidade dos poços amostrados. Estudos visando ao monitoramento e mapeamento de plumas de contaminantes em território brasileiro - cujas condições geológicas e climáticas, bem como de resíduos aterrados, são únicas - são ainda imprescindíveis, de forma a contribuir para o estabelecimento de diretrizes ao gerenciamento dessas áreas.
\end{abstract}

Palavras-Chave: depósito de resíduos, monitoramento, hidrogeoquímica, Aquífero Guarani.

\begin{abstract}
The disposal of solid waste without a proper planning can impact the surroundings for decades or centuries after the activities ended. The impact of the leached contaminants in the surroundings depends on the characteristics of the geological formation, as well as of the occurrence of transport and attenuation processes. This study aimed at studying the contaminants found in the surroundings of an old landfill located in a Guarani Aquifer recharge zone, as well as their seasonal variations. Samples from 14 monitoring wells were collected in July and December 2017 and different parameters were analyzed, such as pH, electrical conductivity, alkalinity, COD, chloride, nitrate, ammoniacal nitrogen, iron, manganese, calcium and metals. High contaminants concentrations were found inside the waste deposit, but a considerable decrease was observed downstream. Even so, a visible influence of the deposit in its surroundings was verified, with the possible occurrence of redox reactions and seasonal quality fluctuations in the sampled wells. Researches focusing on the monitoring and mapping of contaminant plumes in Brazilian territory - whose geological and climatic conditions, as well as landfilled contents, are unique - are still imperative, so as to contribute to the establishment of guidelines for the management of these areas.
\end{abstract}

Keywords: waste deposit, monitoring, hydrogeochemistry, Guarani Aquifer.

\section{INTRODUÇÃO}

Os depósitos de resíduos são fontes de poluição de água subterrânea substanciais, na medida em que podem levar à geração de lixiviados altamente contaminados que percolam pelo subsolo, atingindo os aquíferos e alterando-os por décadas ou séculos, mesmo muito tempo após o término do envio de resíduos e mesmo em se tratando de maciços de pequeno porte (Fetter, 2018).

A grande dificuldade na abordagem e gestão destes locais está na variabilidade da qualidade dos resíduos aterrados, podendo variar de resíduos domésticos a minerais, industriais, de construção e demolição, e até mesmo resíduos perigosos. Isto pode levar à distribuição de diferentes tipos de resíduos de forma aleatória e à consequente geração de plumas de diferentes qualidades. Estas plumas, adicionalmente, têm sua composição alterada com o tempo (Christensen et al., 2001).

Além disto, os mecanismos de transporte e 
atenuação que ocorrem na zona saturada e não saturada circundante aumentam a complexidade dos estudos que visam à previsão do alcance do impacto gerado por estes depósitos.

Assim, uma vez atingindo o aquífero de água subterrânea, os contaminantes passam por processos de diluição, dispersão, reações redox, biodegradação, sorção, complexação, precipitação e troca iônica, levando a mudanças nas concentrações detectadas e a alterações nos formatos e direções das plumas de contaminantes. Ressalta-se que o conjunto destes processos é esperado em maior ou menor grau em todos os aquíferos, mesmo em se tratando de sedimentos arenosos e grosseiros (Bjerg et al., 2013).

É importante enfatizar que o número de plumas geradas por depósitos de resíduos totalmente documentadas é pequeno. Com base nos poucos casos documentados, conduzidos majoritariamente em aquíferos arenosos, se prevê que os processos de transporte e atenuação acima mencionados podem promover uma remediação natural da pluma de contaminantes significante, limitando os efeitos do lixiviado a uma área que não deve exceder $1000 \mathrm{~m}$ a partir do depósito (Christensen et al., 2007).

No entanto, é imprescindível que monitoramentos abrangentes sejam realizados em áreas adjacentes a depósitos de resíduos em diferentes locais, condições geológicas e climáticas, bem como para diferentes qualidades de resíduos aterrados, de forma a permitir a previsão do alcance dos impactos em diferentes contextos. Ressalta-se que, neste âmbito, a adequada localização dos poços é fundamental, de forma a obter amostras relativas à pluma

Este estudo objetiva apresentar resultados de monitoramento da zona saturada adjacente a um antigo depósito de resíduos situado em zona de recarga do Aquífero Guarani. Com isso, visa-se a fornecer subsídios ao entendimento dos processos aos quais os contaminantes estão expostos em áreas similares, auxiliando no estabelecimento de diretrizes para o monitoramento e gerenciamento destes locais, tão abundantes no Brasil.

\section{MATERIAIS E MÉTODOS}

\section{Área de estudo}

O depósito de resíduos Santa Madalena está localizado na região sudeste do perímetro urbano do município de São Carlos-SP, em área de afloramento do Aquífero Guarani, constituída por arenitos da formação Botucatu (PMSC, 2011).

Os solos na área do lixão se enquadram em dois grupos: latossolo vermelho-amarelo, na área de deposição dos resíduos, e areias quartzosas profundas, na região logo adjacente. Os valores de condutividade hidráulica (entre $10^{-4}$ e $10^{-6} \mathrm{~cm} / \mathrm{s}$ ), a granulometria do local (areias finas a médias) e o baixo poder de troca catiônica associado ao solo encontrado levam a uma teórica alta vulnerabilidade do local à contaminação (Gonçalves, 1989; Freitas, 1996; Velozo, 2006).

Com base em levantamento realizado pela PMSC (2011), a direção do fluxo de água subterrânea na região varia em função das estações no ano, sendo que os mapas potenciométricos para julho e dezembro de 2010 foram representados pelos mapas da Figura 1. Similarmente, Matsuzaki (1998) determinou o sentido do fluxo de água subterrânea como NW.

A origem do depósito Santa Madalena está relacionada a uma tentativa de estabilização de uma voçoroca identificada na década de 70 na região, por meio da deposição de resíduos urbanos no

local. Entre 1980 e 1996, a região recebeu resíduos domiciliares, industriais, de construção e demolição e de serviços de saúde, sem qualquer estudo de adequabilidade e sem a implantação de estruturas de proteção das águas subterrâneas e da saúde pública. Pelo contrário, os resíduos foram localizados abaixo do nível de água subterrânea (Velozo, 2006). Este conjunto de fatores levou o local a fazer parte da lista de áreas contaminadas do Estado de São Paulo, segundo a CETESB (2017).

Tendo em vista o potencial de contaminação que este depósito oferece ao entorno e sua importância para os mananciais de abastecimento do município de São Carlos, diversos estudos foram realizados no local (Gonçalves, 1986; Rios, 1993; Bossolan, 1993; Menezes, 1995; Freitas, 1996; Gadotti, 1997; Matsuzaki, 1998; Álvares, 2000; Velozo, 2006; PMSC, 2011; Shinzato, 2014; Pelinson, 2018).

Estes estudos apontam que o lixão Santa Madalena ainda é uma grande fonte poluente, mas que a atenuação e o transporte no meio ainda são pouco entendidos. Há certa controvérsia quanto à possível existência de formações geológicas que auxiliem na proteção do aquífero profundo (Álvares, 2000) ou, pelo contrário, à existência de uma descontinuidade abaixo do depósito que pode levar à contaminação do lençol (Velozo, 2006). 


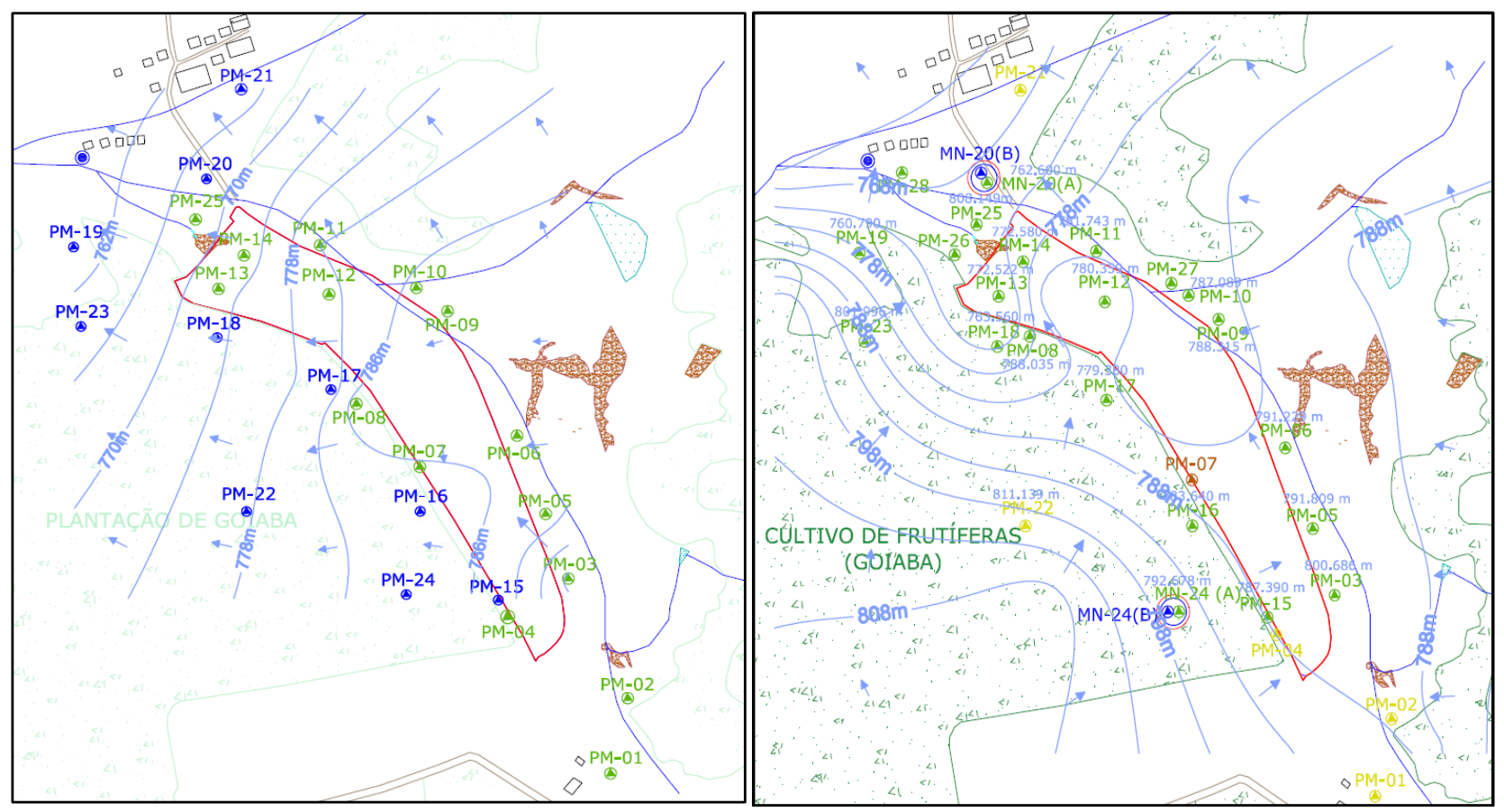

Figura 1 - Mapas potenciométricos da área de estudo, em julho/2010 (esquerda) e dezembro/2010 (direita). O depósito de resíduos apresenta-se delimitado pela linha vermelha. FONTE: PMSC, 2011.

Ressalta-se que, com base na solubilização dos resíduos amostrados em diferentes profundidades, Shinzato (2014) apontou que estes ainda não podem ser considerados inertes, e que as regiões de acúmulo de lixiviado no depósito podem ser liberadas ao meio com o tempo ou com a incidência de eventos climáticos extremos, levando ao fluxo de contaminantes para o entorno.

\section{Coleta e análise físico química de amostras de} água subterrânea

Foram coletadas amostras de água subterrânea em 14 poços de monitoramento existentes no interior (P14, EM, P15 e P18), a montante (P12, $\mathrm{P} 13$ e P23) e a jusante do depósito de resíduos (P2, P3, P7, P9, P10, P16 e P17), como ilustra a Figura
2.

As coletas foram realizadas em julho/2017 e dezembro/2017, de forma a representar a sazonalidade do local.

As amostras foram coletadas e analisadas em conformidade com o manual Standard Methods for the Examination of Water and Wastewater (APHA, 2012). As seguintes análises foram realizadas: $\mathrm{pH}$, condutividade (EC), potencial redox (Eh), alcalinidade, nitrito, nitrato, nitrogênio amoniacal, fosfato, fluoreto, cloreto, demanda química de oxigênio (DQO) e os metais alumínio, antimônio, bário, cádmio, cálcio, chumbo, cobalto, cobre, cromo, estrôncio, ferro, magnésio, manganês, níquel, potássio, prata, selênio, sódio e zinco.

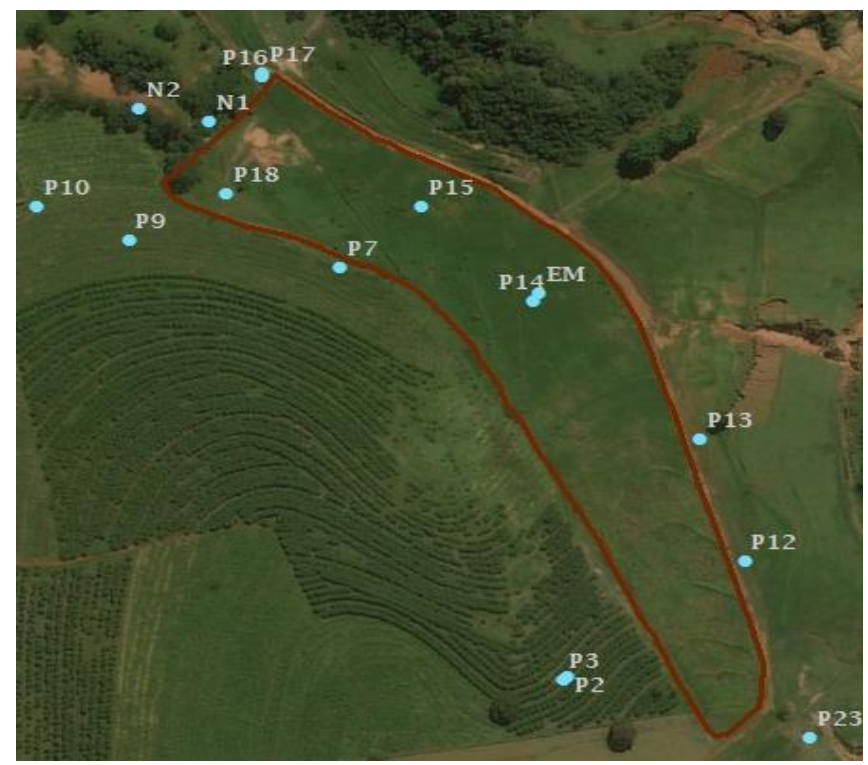

Figura 2 - Localização dos poços de monitoramento amostrados. 


\section{RESULTADOS E DISCUSSÃO}

São apresentados nas Tabelas 1 e 2 os principais resultados das análises realizadas. Os parâmetros estão em $\mathrm{mg} / \mathrm{L}$, com exceção do $\mathrm{pH}$ intervenção da Decisão de Diretoria $n^{\circ}$ 256/2016 da CETESB (VI) e os padrões de potabilidade da (sem unidade), da condutividade (EC, em $\mu \mathrm{S} / \mathrm{cm}$ ) e do potencial redox $(\mathrm{Eh}, \mathrm{em} \mathrm{mV})$. Os valores de Portaria $n^{\circ} 2914$ de 2011 (PP) também são apresentados, sendo que os valores acima destes padrões são destacados em vermelho.

Tabela 1 - Parâmetros físico-químicos das amostras de água subterrânea: julho/2017

\begin{tabular}{|c|c|c|c|c|c|c|c|c|c|c|c|c|c|c|}
\hline PARAMMETRO & $P 7$ & $P 9$ & $P 10$ & $P 12$ & $P 13$ & $P 17$ & $P 2$ & $P 3$ & $P 14$ & P 15 & P 18 & $P 23$ & $V I^{*}$ & $P P * *$ \\
\hline Temperatura $\left({ }^{\circ} \mathrm{C}\right)$ & 17,37 & 16,44 & 17,54 & 18,24 & 18,36 & 17,66 & & & 17,33 & 17,21 & 16,57 & & & \\
\hline$E h(m V)$ & 231,0 & 242,1 & 243,8 & $-51,4$ & 254,0 & 274,1 & 212,2 & 175,7 & 3,9 & 128,1 & 184,2 & & & \\
\hline$p H$ & 5,2 & 4,8 & 4,8 & 6,3 & 5,1 & 5,2 & 5,3 & 6,4 & 7,0 & 6,2 & 5,3 & 5,5 & & \\
\hline $\begin{array}{c}\text { Condutividade } \\
(\mu \mathrm{S} / \mathrm{cm})\end{array}$ & 79,1 & 49,2 & 34,6 & 543,0 & 26,0 & 34,9 & 18,7 & 56,4 & 1026,0 & 120,5 & 414,0 & 40,6 & & \\
\hline$N . A .(m)$ & 7,14 & 15,10 & 13,15 & 19,97 & 14,26 & 8,92 & 27,64 & 28,61 & 2,85 & 5,77 & & & & \\
\hline Alcalinidade $(m g / L)$ & 3,2 & 0,0 & 0,0 & 244,0 & 7,0 & 8,8 & 31,2 & 46,8 & 66,5 & 8,1 & 19,6 & 11,9 & & \\
\hline $\mathrm{N}-\mathrm{NO}_{2}^{-}(\mathrm{mg} / \mathrm{L})$ & 0,010 & 0,020 & 0,020 & 0,005 & 0,005 & 0,007 & 0,008 & 0,005 & 0,026 & 0,002 & 2,010 & 0,004 & & 1,000 \\
\hline $\mathrm{N}-\mathrm{NO}_{3}^{-}(\mathrm{mg} / \mathrm{L})$ & 4,50 & 3,28 & 1,79 & 1,24 & 1,11 & 0,95 & 5,59 & 1,48 & 3,14 & 0,38 & 0,01 & 0,71 & 10,00 & 10,00 \\
\hline$N-N H_{j}(m g / L)$ & 0,06 & 0,06 & 0,02 & 0,58 & 0,01 & 0,09 & 1,96 & 0,08 & 46,25 & 1,67 & 2,55 & 0,11 & & 1,5 \\
\hline$C t(m g / L)$ & 6,4 & 3,4 & 2,4 & 9,2 & 1,0 & 2,4 & 2,1 & ND & 27,0 & 2,6 & 105,0 & 1,1 & & 250 \\
\hline$D Q O(m g / L)$ & 13 & 14 & 15 & 23 & 13 & 15 & 35 & 16 & 62 & 14 & 6 & 19 & & \\
\hline $\mathrm{PO}_{f^{-}}(\mathrm{mg} / \mathrm{L})$ & 0,04 & 0,01 & 0,01 & 0,07 & 0,06 & 0,49 & 0,01 & 0,17 & 0,07 & 0,20 & 0,03 & 0,06 & & \\
\hline$P b(m g / L)$ & <branco & $\angle$ branco & $\angle$ branco & $<$ branco & \&branco & $<$ branco & 0,02 & 0,01 & $\angle$ branco & 0,04 & $\angle$ branco & $\angle$ branco & 0,01 & 0,01 \\
\hline$M n(m g / L)$ & 0,025 & \&branco & <branco & 1,302 & $<$ branco & $<$ branco & 0,385 & <branco & 0,986 & 0,237 & 0,368 & $<$ branco & & 0,1 \\
\hline$F e(m g / L)$ & \&branco & 0,091 & 0,194 & 8,492 & 0,210 & 0,473 & 0,006 & <branco & 0,032 & 10,872 & 23,340 & 0,499 & & 0,300 \\
\hline$C d(m g / L)$ & <branco & <branco & 0,020 & $\angle$ branco & 0,016 & Zbranco & $\angle$ branco & $\angle$ branco & <branco & <branco & 0,015 & 0,015 & 0,005 & 0,005 \\
\hline $\mathrm{Ca}(\mathrm{mg} / \mathrm{L})$ & 1,377 & 2,017 & 1,847 & 36,987 & 1,769 & 2,177 & 2,307 & 4,567 & 74,887 & 122,487 & 7,149 & 1,869 & & \\
\hline
\end{tabular}

Tabela 2 - Parâmetros físico-químicos das amostras de água subterrânea: dezembro/2017.

\begin{tabular}{|c|c|c|c|c|c|c|c|c|c|c|c|c|c|c|c|}
\hline PARÂMETRO & P7 & $P 9$ & P 10 & $P 11$ & $P 12$ & $P 13$ & $P 16$ & $P 17$ & $P 2$ & $P 3$ & $P 14$ & $P 15$ & $P 18$ & $V I^{*}$ & $P P * *$ \\
\hline Temperatura $\left({ }^{\circ} \mathrm{C}\right)$ & 17,32 & 17,28 & 16,69 & 17,24 & 17,48 & 16,83 & 17,01 & 18,32 & 18,32 & 17,78 & 18,08 & 17,27 & 17,21 & & \\
\hline$E h(m V)$ & 313,2 & 284,0 & 329,4 & 294,1 & 14,2 & 137,9 & 217,0 & 158,0 & 158,0 & 161,1 & 172,8 & 97,8 & 301,7 & & \\
\hline pH & 6,3 & 6,3 & 5,9 & 6,6 & 8,0 & 7,7 & 6,1 & 7,7 & 7,7 & 8,3 & 8,6 & 7,8 & 6,0 & & \\
\hline Condutividade $(\mu \mathrm{S} / \mathrm{cm})$ & 166,7 & 53,8 & 38,8 & 138,3 & 506,0 & 32,2 & 149,6 & 40,1 & 103,4 & 65,0 & 1195,0 & 120,3 & 314,0 & & \\
\hline N.A. $(m)$ & 8,27 & 15,54 & 13,42 & 16,32 & 20,11 & 14,57 & 5,69 & 9,13 & 27,64 & 28,64 & 6,25 & 6,16 & 7,23 & & \\
\hline Alcalinidade(mg/L) & 2,1 & 1,1 & 0,0 & 13,3 & 230,3 & 8,4 & 0,0 & 10,9 & 0,0 & 26,3 & 57,1 & 48,0 & 0,7 & & \\
\hline $\mathrm{N}-\mathrm{NO}_{2}^{-}(\mathrm{mg} / \mathrm{L})$ & 0,007 & 0,004 & 0,003 & 0,003 & 0,008 & 0,004 & 0,004 & 0,004 & 0,026 & 0,008 & 0,075 & 0,004 & 0,007 & 1,000 & \\
\hline $\mathrm{N}-\mathrm{NO}_{3} \cdot(\mathrm{mg} / \mathrm{L})$ & 6,45 & 2,88 & 1,64 & 6,33 & 1,17 & 2,04 & 10,84 & 2,81 & 5,85 & 2,26 & 10,88 & 0,90 & 2,53 & 10,00 & \\
\hline $\mathrm{N}-\mathrm{NH}_{4}(\mathrm{mg} / \mathrm{L})$ & 0,24 & 0,01 & 0,00 & 0,00 & 0,40 & 0,03 & 0,02 & 0,10 & 1,39 & 0,15 & 45,25 & 1,66 & 1,98 & & 1,50 \\
\hline $\mathrm{Cl} \cdot(\mathrm{mg} / \mathrm{L})$ & 11,4 & 4,9 & 3,7 & 14,7 & 6,3 & 1,3 & 10,7 & 2,0 & 10,0 & 1,8 & 65,0 & 4,2 & 69,0 & & 250,0 \\
\hline$D Q O(m g / L)$ & 59 & 21 & 16 & 17 & 20 & 36 & 16 & 18 & 25 & 60 & 122 & 23 & 35 & & \\
\hline $\mathrm{PO}_{4}{ }^{3-}(\mathrm{mg} / \mathrm{L})$ & 0,08 & 0,02 & 0,02 & 0,03 & 0,05 & 0,14 & 0,04 & 0,51 & 0,03 & 0,20 & 0,05 & 0,07 & 0,03 & & \\
\hline $\mathrm{Pb}(\mathrm{mg} / \mathrm{L})$ & $<$ branco & <branco & $<$ branco & $<$ branco & $<$ branco & $<$ branco & $<$ branco & $<$ branco & $<$ branco & 0,03 & 0,05 & $<$ branco & $<$ branco & 0,01 & 0,01 \\
\hline$M n(m g / L)$ & 0,299 & 0,025 & 0,007 & $<$ branco & 1,191 & $<$ branco & 1,086 & $<$ branco & 0,595 & 0,116 & 0,271 & 0,059 & 0,360 & & 0,1 \\
\hline $\mathrm{Fe}(\mathrm{mg} / \mathrm{L})$ & 0,140 & $<$ branco & 0,050 & 0,133 & 3,505 & 0,387 & 0,153 & 0,671 & $<$ branco & 0,321 & 0,416 & 3,125 & 5,890 & & 0,300 \\
\hline$C d(m g / L)$ & 0,002 & 0,005 & 0,005 & 0,003 & $<$ branco & 0,006 & 0,010 & 0,007 & 0,006 & 0,005 & 0,006 & 0,009 & 0,007 & 0,005 & 0,005 \\
\hline $\mathrm{Ca}(\mathrm{mg} / \mathrm{L})$ & 3,038 & 1,988 & 1,438 & 10,188 & 47,288 & 4,508 & 8,038 & 7,738 & 3,228 & 6,288 & 66,000 & 6,108 & 9,133 & & \\
\hline
\end{tabular}

Da análise das tabelas, destacam-se as seguintes observações: a. Os poços de monitoramento localizados no interior do maciço (P14, P15 e P18) são 
aqueles que apresentam maiores concentrações de contaminantes, destacando-se os elevados valores de condutividade, alcalinidade, nitrogênio amoniacal, cloretos, ferro e manganês. As anomalias encontradas condizem com os parâmetros característicos de lixiviados e áreas impactadas por estes (Kjeldsen et al., 2002; Christensen et al., 2001; Paschoalato et al., 2001), mostrando significativa influência do depósito na formação geológica existente em sua base.

b. Os poços localizados a jusante do maciço mostram a influência deste, com a detecção de diferentes contaminantes, no entanto sua concentração reduz consideravelmente em relação àquelas detectadas no interior do depósito. Este resultado condiz com Elis \& Zuquette (2002), cujo estudo foi condizido em condições semelhantes às do presente trabalho. Avaliando os impactos gerados por um depósito irregular de resíduos (uma voçoroca aterrada com resíduos sólidos urbanos e industriais) situado no município de Ribeirão Preto-SP, em área de recarga do Aquífero Guarani (formação Botucatu), os autores encontraram baixas concentrações de contaminantes nos poços de entorno. Similarmente, diversos estudos conduzidos em aquíferos arenosos e citados por Christensen et al. (2000) mostraram que as plumas de contaminantes não costumam exceder os $1 \mathrm{~km}$ de extensão. Outros estudos, no entanto, mostram plumas oriundas de depósitos de resíduos atingindo distâncias maiores que $5 \mathrm{~km}$ (Chakraborty et al., 2016; Rana et al., 2017).

c. O poço P12, localizado fora do depósito e contra o fluxo previsto da água subterrânea (vide Figura 1), apresentou, em todas as coletas, visíveis anomalias: valores de Eh negativos, altos valores de condutividade e alcalinidade, bem como de ferro e manganês. Isto pode ser explicado por: a) ocorrência de mudanças no sentido do fluxo de águas subterrâneas com as variações sazonais; b) anomalias no fluxo subterrâneo, o que é previsto em áreas de depósito de resíduos, pois, devido às variações de condutividade hidráulica existentes no interior no maciço e à entrada de água pluvial no mesmo, pode haver aumento da carga hidráulica no interior deste e direcionamento do fluxo para as laterais (Belevi \& Baccini, 1989; Christensen et. al., 2000); ou c) à existência de resíduos em locais não previstos pela delimitação inicial do depósito, ou seja, na região do poço P12. Desta maneira, é importante concluir que poços de monitoramento devem ser instalados em todo o entorno de depósitos de resíduos, de forma a assegurar a inexistência de contaminação, especialmente em locais de disposição irregular.

d. Atenção deve ser dada aos valores de chumbo e de cádmio acima dos limites de intervenção da Decisão de Diretoria $n^{\circ}$ 256/2016/E da CETESB $(0,01 \mathrm{mg} / \mathrm{L}$ para chumbo e $0,005 \mathrm{mg} / \mathrm{L}$ para cádmio) para os poços $2,10,13,14,15,16,17,18$ e 23 . Ressalta-se que metais potencialmente tóxicos não são consideravelmente móveis em condições naturais de $\mathrm{pH}$ e $\mathrm{Eh}$, e sofrem considerável atenuação no meio. Altas concentrações de metais não são, portanto, previstas mesmo a pequenas distâncias da fonte poluente, especialmente em se tratando de depósito de resíduos majoritariamente urbanos. Desta maneira, as concentrações altas de chumbo e cádmio podem estar associadas a maiores contribuições de resíduos industriais e de demolição existentes no depósito, o que deve ser cuidadosamente caracterizado e monitorado de forma a evitar riscos à saúde pública. Altas concentrações de metais também foram encontradas em depósito irregular em Franca-SP (Gilberto, 2007), onde resíduos industriais e de curtume também foram descartados sem qualquer proteção e prevenção da poluição. É importante ressaltar que não há contribuição da matriz geológica na concentração destes metais, uma vez que não se prevê a existência de minerais de cádmio e chumbo na região (CETESB, 2016). Finalmente, ressalta-se que os baixos valores de $\mathrm{pH}$ comumente encontrados em áreas livres e rasas do aquífero Botucatu (CETESB, 2016) podem levar a uma maior mobilidade de metais potencialmente tóxicos (Fetter, 2018), o que pode parcialmente justificar a detecção destes em poços de monitoramento do entorno.

e. Sob o ponto de vista geoquímico, é importante ressaltar as altas concentrações de cálcio detectadas em vários poços, especialmente nos números 12, 14 e 15, mostrando que pode estar havendo dissolução mineral ou troca catiônica em decorrência do impacto do contato do lixiviado com a formação geológica local. Também pode haver contribuição de resíduos de construção e demolição na pluma de contaminantes. Nematollahi et al. (2018) 
também justificaram as altas concentrações de cálcio detectadas em alguns poços de monitoramento pela dissolução de minerais como calcita, aragonita, dolomita, e minerais de silicatos, ou pela ação antrópica.

f. A presença de ferro e manganês está associada à ocorrência de reações redox na área de influência, com a oxidação da matéria orgânica proveniente do lixiviado levando à depleção de formas oxidadas presentes no meio (Christensen, 1997). Dessa maneira, maiores concentrações de ferro e manganês estão associadas à redução de formas mais oxidadas $\left(\mathrm{Fe}^{3+}\right.$ e $\left.\mathrm{Mn}^{4+}\right)$ para formas reduzidas $\left(\mathrm{Fe}^{2+} \mathrm{e} \mathrm{Mn}\right.$ $\left.{ }^{2+}\right)$, as quais são mais móveis e solúveis. Por conseguinte, a detecção de maiores concentrações de ferro e manganês está relacionada com a degradação da matéria orgânica existente na pluma de contaminantes (vide dados dos poços P12, P15, P18 e P16).

g. Em relação às concentrações de cloretos, estas são usadas como referências na delimitação da pluma de contaminação derivada de depósito de resíduos, uma vez que, sendo geradas na fonte poluente, não sofrem quaisquer processos de atenuação, mas apenas transporte e diluição (Cook \& Herczeg, 2000). Desta maneira, ainda que este composto não tenha importância sob o ponto de vista de saúde pública, sua detecção é importante na medida em que mostra aqueles pontos que estão sendo influenciados mais significativamente pela pluma, e que, portanto, podem apresentar outros compostos de maior risco ambiental.

Com a finalidade de possibilitar a melhor visualização da distribuição espacial das concentrações na área de estudo, foram construídos mapas de distribuição de alguns parâmetros de importância para áreas contaminadas por depósitos de resíduos (condutividade elétrica, cloretos, alcalinidade e ferro) para os valores médios dos diferentes parâmetros.

Os mapas construídos são apresentados nas Figuras 3, 4, 5 e 6. Pela análise dos mapas, e reiterando o que já foi discutido anteriormente, se verifica que existe grande alteração dos parâmetros avaliados no interior do depósito de resíduos (P14, P15 e P18), mas significativa atenuação no entorno deste (P7, P9, P10, P16, P17, P2 e P3). Este comportamento foi verificado em diversos estudos em áreas impactadas por depósitos de resíduos (Rana et al., 2017; Van Breukelen \& Griffioen, 2004; Elis \& Zuquette, 2002; Christensen et al, 2000; Gadotti, 1997), tendo sido muitas vezes atribuído a uma capacidade de auto-limpeza ou autodepuração dos contaminantes (Menezes, 1995; Przydatek \& Kanownik, 2019).

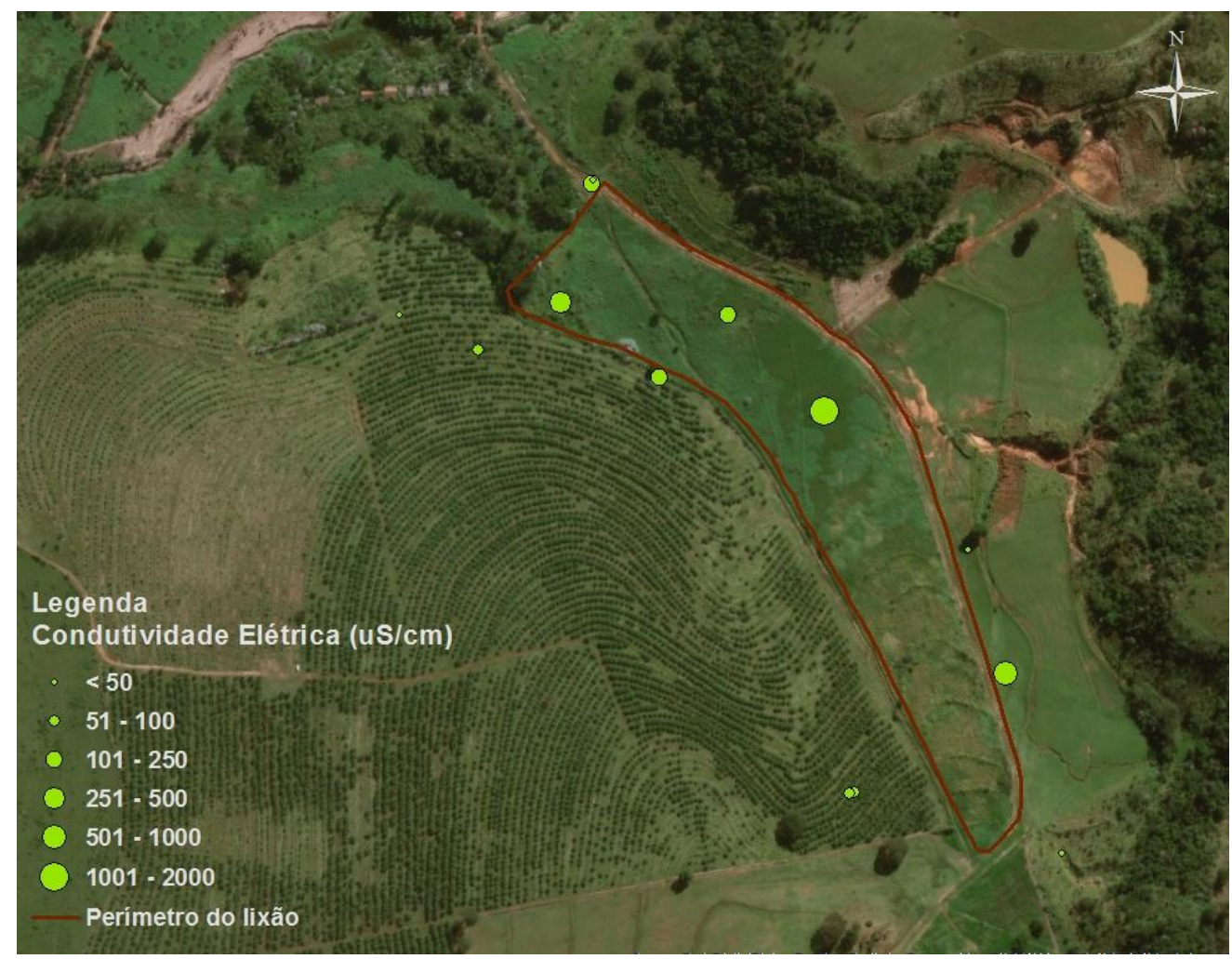

Figura 3 - Variação espacial dos valores médios de condutividade elétrica encontrados em 2017. 


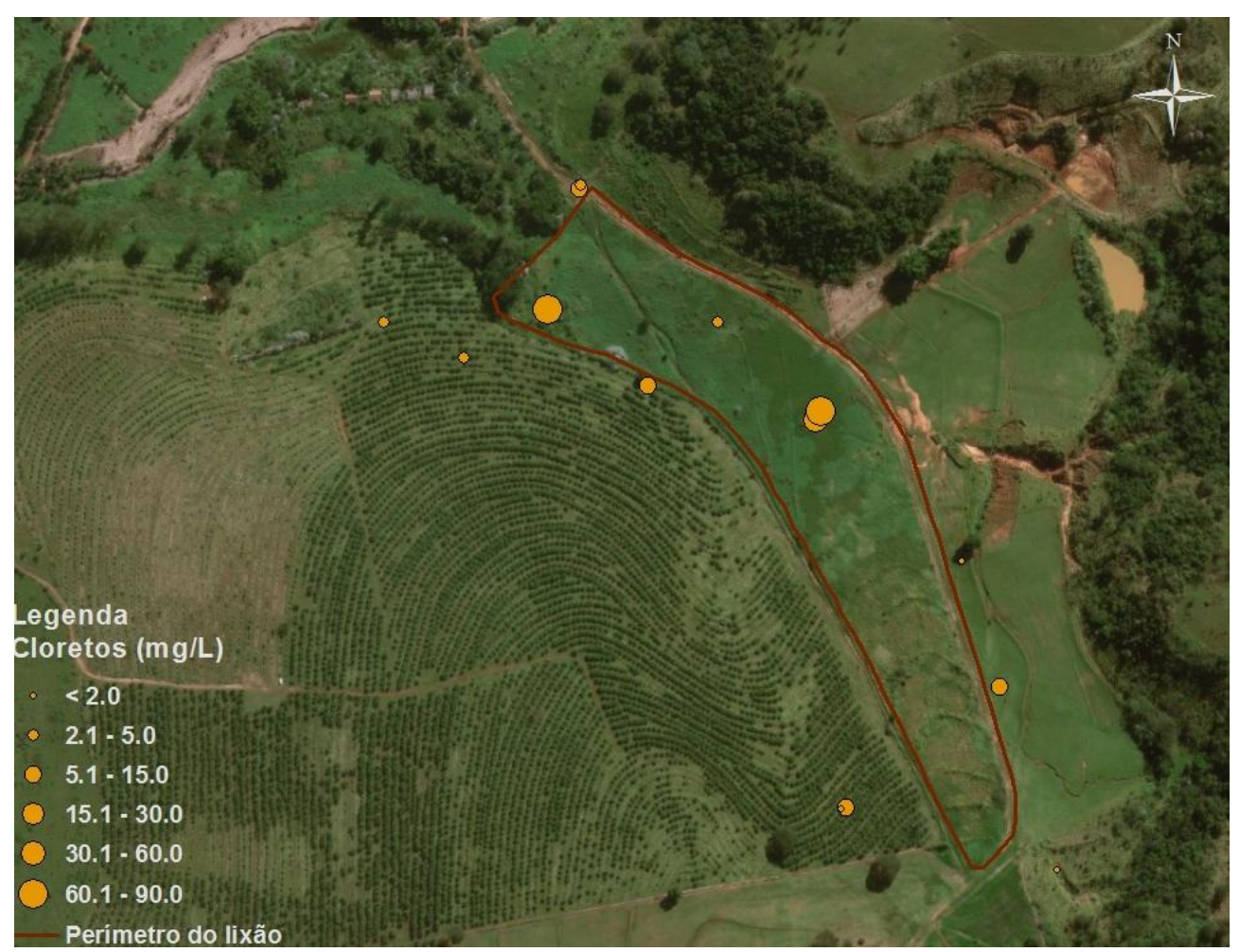

Figura 4 - Variação espacial dos valores médios de concentração de cloretos encontrados em 2017.

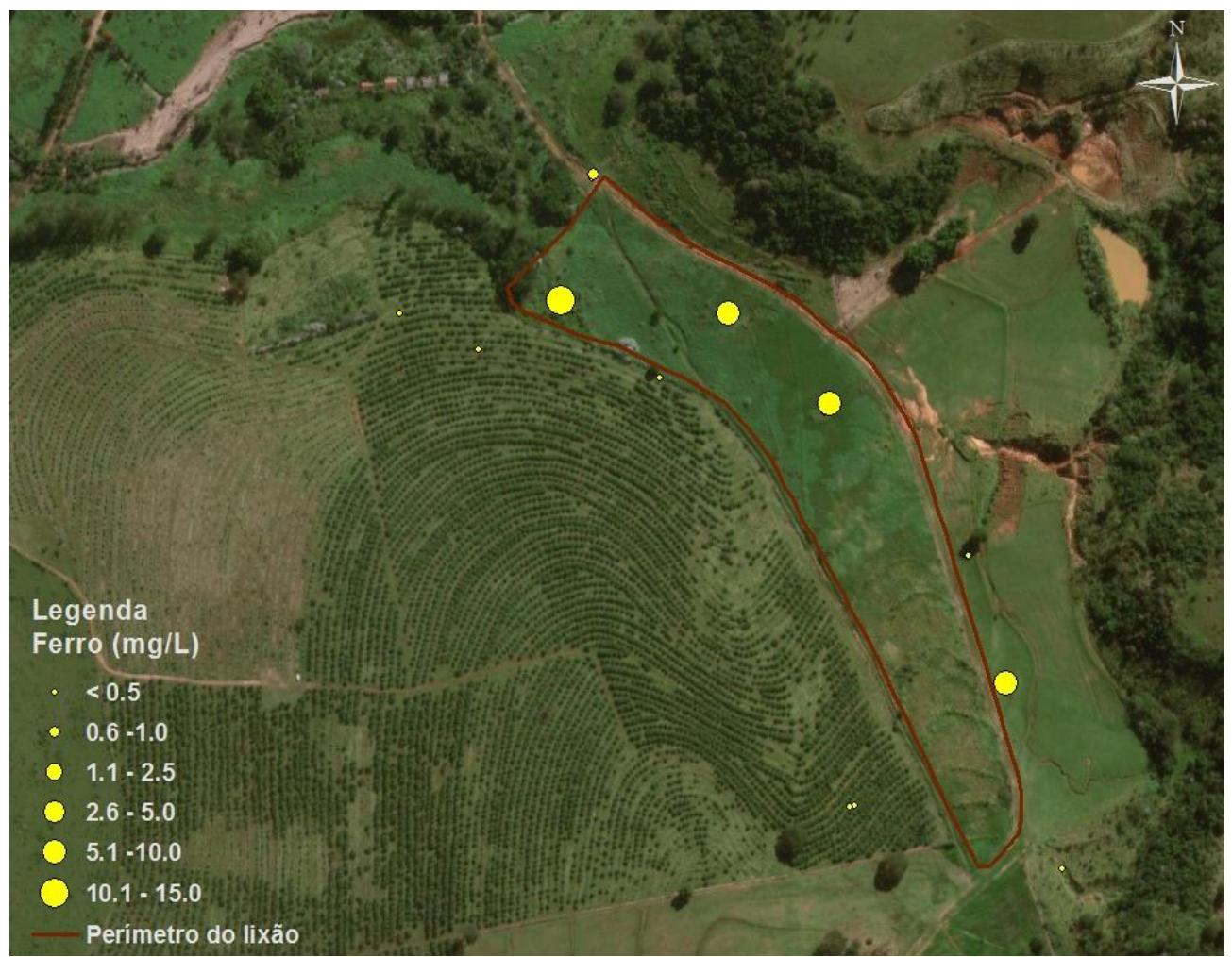

Figura 5 - Variação espacial dos valores médios de concentração de ferro encontrados em 2017.

A explicação para este comportamento, no entanto, pode ser atribuída a diversos fatores. Por um lado, a capacidade de atenuação da formação geológica pode ser maior do que a esperada, devido à presença de óxidos e hidróxidos que aumentam a capacidade de sorção do meio, no caso da Formação Botucatu (Fagundes \&
Zuquette, 2011), e levam a uma redução do alcance da pluma.

Por outro lado, as velocidades de fluxo da água subterrânea devem ser consideradas, uma vez que, sendo reduzidas (aproximadamente de 0,5 m/ano na região de estudo, segundo Sindico et al., 2018) devem produzir uma área de influência do aterro 
restrita, tendo em vista o periodo estudado (40 anos desde o início da deposição). Neste último caso, supõe-se que a pluma de contaminantes ganharia maiores extensões no decorrer dos próximos anos, levando a um aumento nas concentrações verificadas em diferentes poços, o que deve ser adequadamente monitorado. Esta última hipótese está de acordo com o sugerido por Rana et al. (2017).

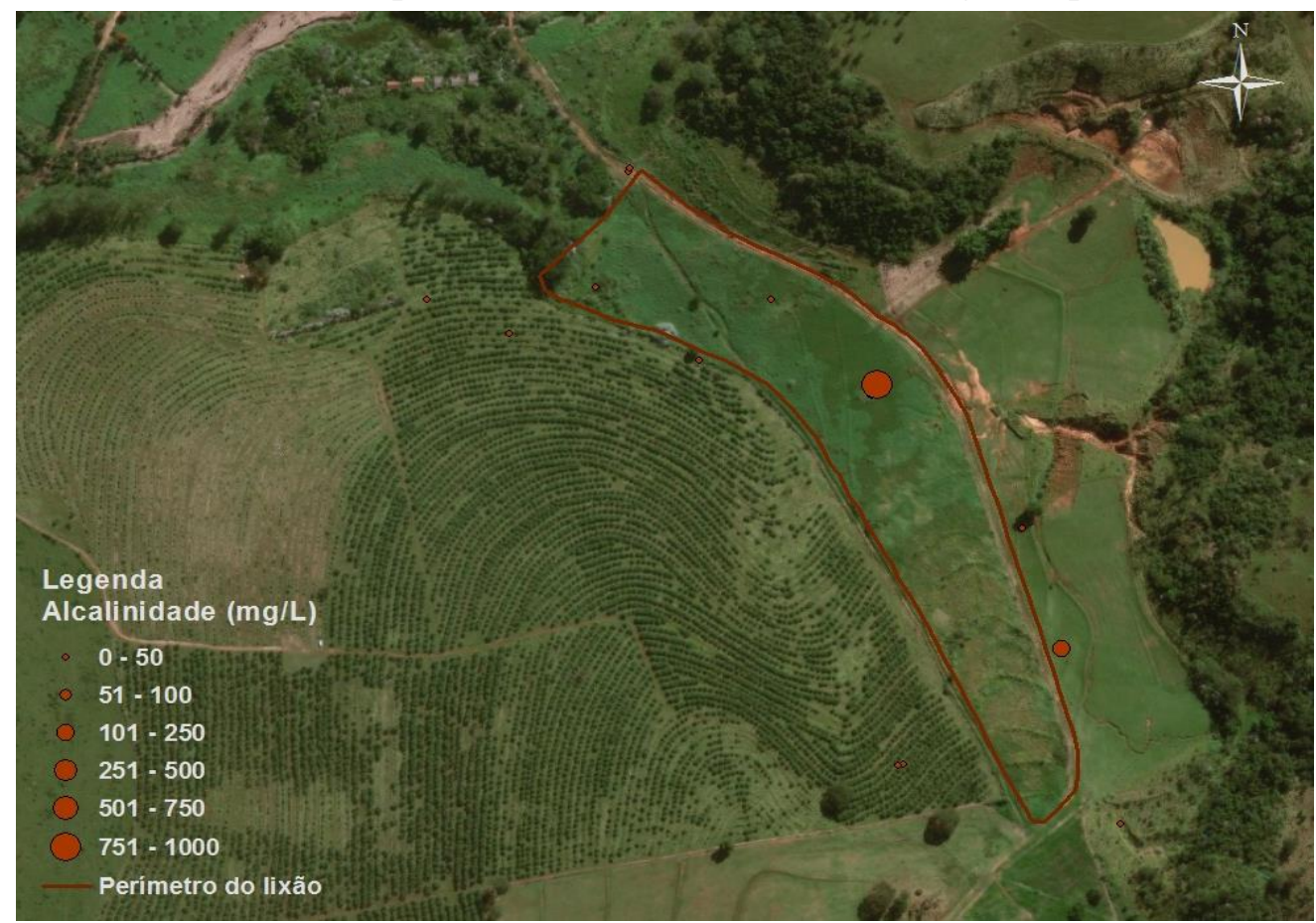

Figura 6 - Variação espacial dos valores médios de alcalinidade encontrados em 2017.

Ainda com base nas Figuras de 3 a 6, também é possível verificar claramente a zona de influência do depósito de resíduos, ainda que sejam verificadas baixas concentrações dos contaminantes. É chamativo, mais uma vez, o comportamento do poço P12, o qual se supunha estar fora da área de influência do depósito, mostrando clara anomalia hidrogeológica em áreas afetadas por depósitos de resíduos, o que condiz com o exposto por Christensen et al (2000).

\section{Diagramas de Piper}

Os dados provenientes das amostras coletadas permitiram a construção de diagramas de Piper, visando à classificação das águas locais e à detecção de anomalias. Os diagramas são apresentados nas Figuras 7 e 8.

Pela análise dos diagramas de Piper, se verifica que a maioria das amostras sofreu mudanças quanto à classificação em decorrência da sazonalidade, com exceção do poço P9, cujas águas permaneceram classificadas como mistas cloretadas em todas as coletas.

Nas coletas realizadas em julho/2017, predominaram as águas bicarbonatadas ( 9 amostras em ambas as coletas, contra apenas 3 amostras cloretadas e nenhuma sulfatada), enquanto em dezembro/2017 predominaram as águas cloretadas
(8 amostras contra 5 bicarbonatadas).

Conclui-se, desta avaliação inicial, que as águas subterrâneas da região são predominantemente bicarbonatadas, o que é característica do aquífero Botucatu (Silva, 1983). A ocorrência de águas cloretadas esteve associada à estação mais chuvosa, o que pode ser explicado pela maior intensidade dos processos de lixiviação do depósito com a chuva, causando o transporte de cloretos - parâmetro característico de plumas oriundas de depósitos de resíduos - aos poços do entorno. Este fenômeno foi bastante visível para os poços P2, P7, P9, P10, $\mathrm{P} 14$, P18 e P16, os quais situam-se no interior ou a jusante do depósito de resíduos.

Em relação à proporção de cátions, se verificou que a classificação das águas também variou com a sazonalidade, sendo que em julho/2017 predominaram as águas cálcicas, e em dezembro/2017 predominaram as águas mistas. No caso das águas mistas, não se verifica maior expressividade de algum cátion, não sendo possível estabelecer conclusões quanto à influência do depósito de resíduos. A alteração na classificação com a sazonalidade, no entanto, mostra indício de carreamento de íons do depósito e/ou dissolução mineral. 


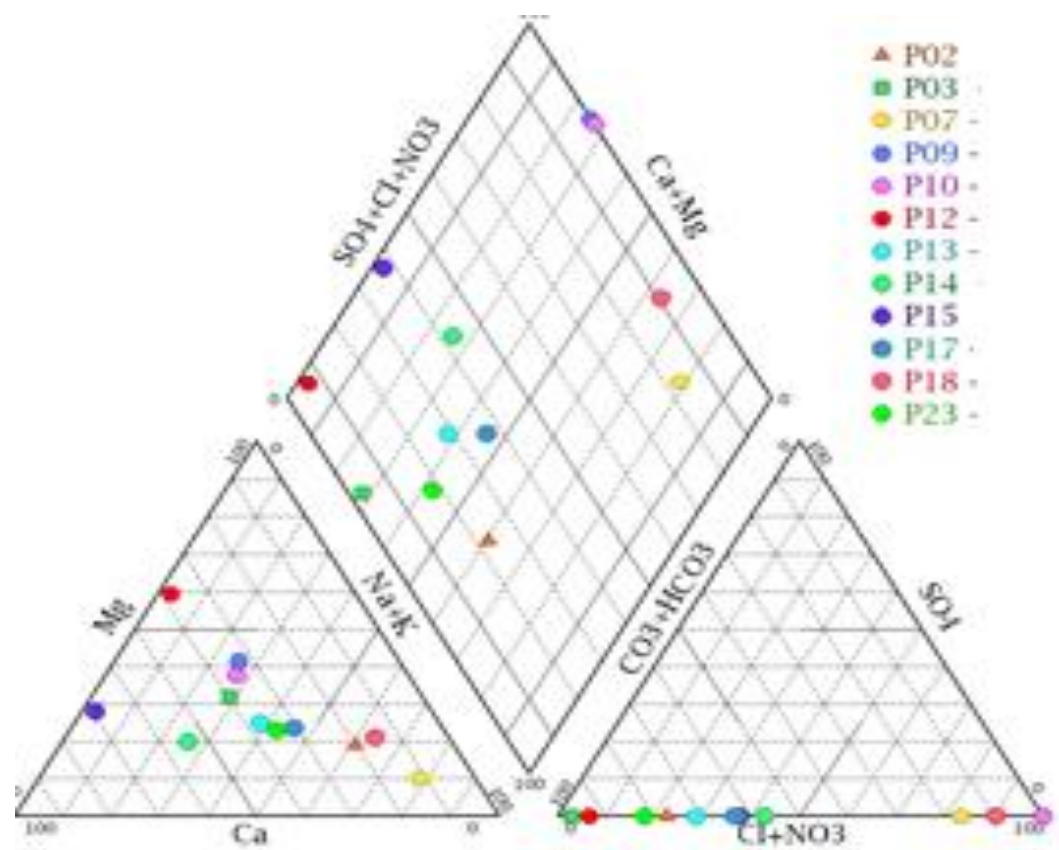

Figura 7 - Diagrama de Piper para as amostras coletadas em julho/2017.

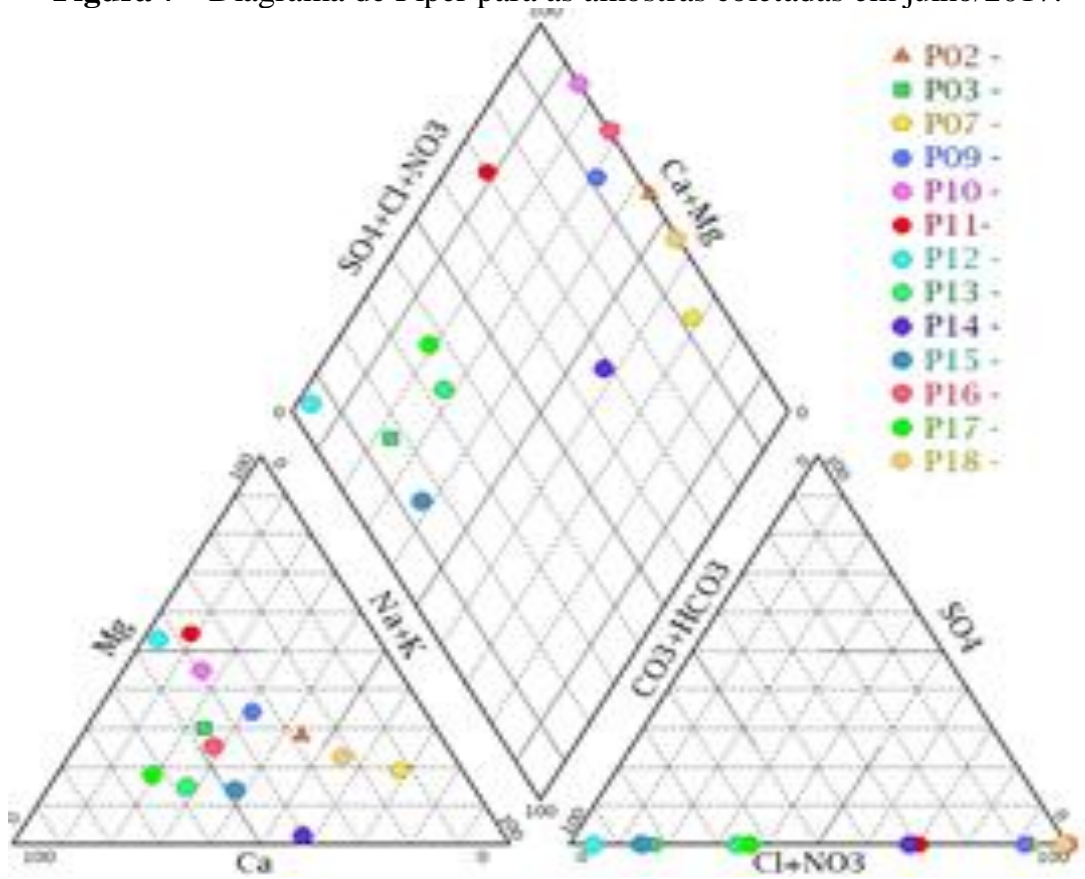

Figura 8 - Diagrama de Piper para as amostras coletadas em dezembro/2017.

\section{Análise de Componentes Principais}

Uma vez que as amostras de água subterrânea formam um conjunto de dados muito amplo e de difícil análise, se optou por realizar a análise estatística de componentes principais (ACP ou PCA, do inglês Principal Component Analysis), de maneira a reduzir o número de variáveis e entender os parâmetros de maior importância para o conjunto de dados. A análise dos dados das diferentes coletas foi feita separadamente, sendo que em todos os casos se obteve uma variância de cerca de $60 \%$ para os fatores F1 e F2, o que foi considerado suficiente para esta pesquisa.

Pela análise dos gráficos, se verifica que houve variabilidade na distribuição dos parâmetros para as distintas coletas, o que era esperado uma vez que os fatores são calculados diferentemente para cada conjunto de dados. No entanto, verificam-se algumas semelhanças, como a importância dos parâmetros condutividade, DQO, nitrogênio amoniacal e fluoretos para o componente $\mathrm{F} 1$, e dos parâmetros alcalinidade, fosfato, nitrito, cloretos e ferro para o componente F2 (com algumas variações). Isto mostra que estes parâmetros identificados são responsáveis por aproximadamente $60 \%$ da variabilidade entre as amostras, o que é condizente com os parâmetros de maior importância para áreas influenciadas 
por depósitos de resíduos sugeridos por Christensen etal. (2001), Paschoalato et al (2001) e Kjeldsen et al (2002).

Em relação à localização das amostras nos gráficos, verifica-se que os poços P14 e EM são caracterizados quase completamente pelo fator F1. Os poços P12 e P18 se localizam, na maioria dos casos, nos lados positivos dos fatores F1 e F2, estando positivamente relacionados com eles. Os demais poços estão majoritariamente aglomerados no terceiro quadrante do gráfico, apresentando, portanto, baixos valores dos parâmetros de F1 e F2 e, portanto, baixas concentrações dos contaminantes ponderados.

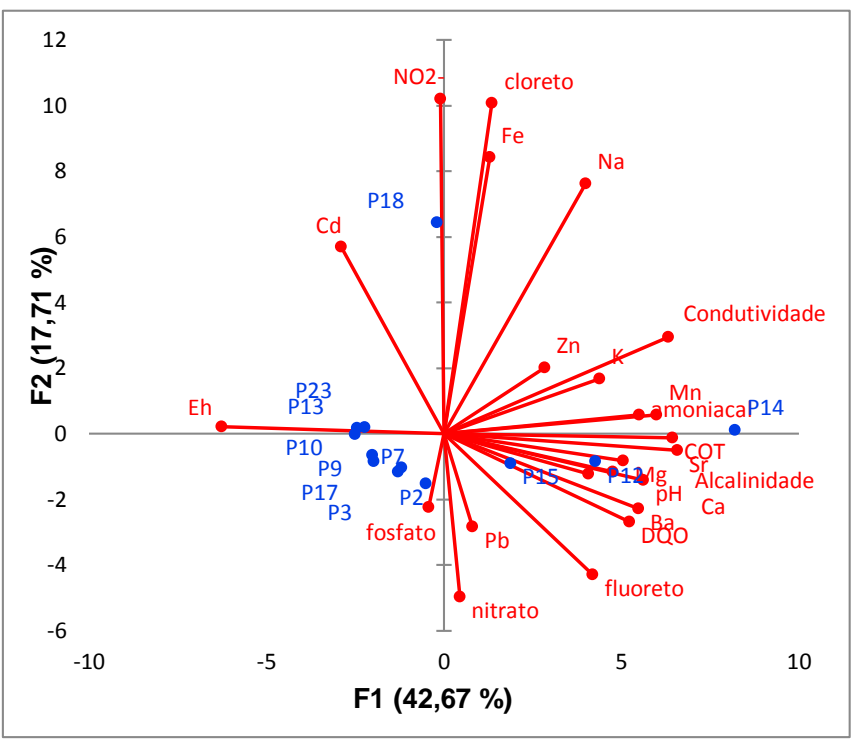

Figura 9 - Análise de componentes principais para o conjunto de dados de julho/2017.

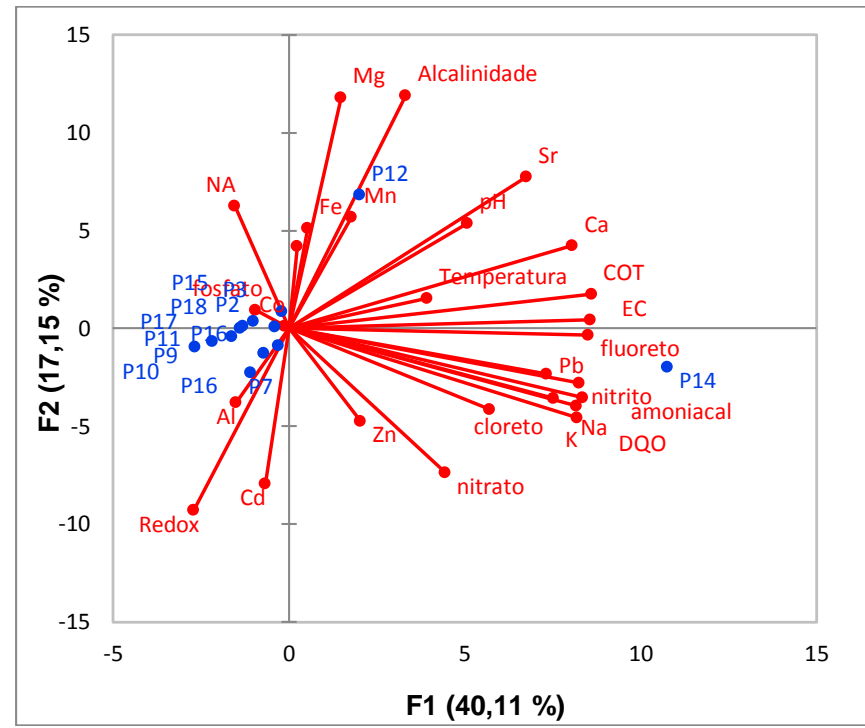

Figura 10 - Análise de componentes principais para o conjunto de dados de dezembro/2017.

\section{CONCLUSÕES}

Este trabalho permitiu observar os impactos que um antigo depósito de resíduos ainda fornece ao entorno, em uma área de recarga do Aquífero Guarani (Formação Botucatu). Com base nos dados coletados, há indícios de atenuação dos contaminantes pela formação geológica - apesar de sua baixa capacidade teórica. Isto condiz com o exposto por Fagundes \& Zuquette (2011), os quais reiteram que, mesmo estando associada a uma baixa capacidade de atenuação, a formação
Botucatu possui óxidos e hidróxidos que aumentam significativamente a sorção de contaminantes, especialmente quando trata-se de baixas concentrações.

No entanto, ainda que haja redução significativa das concentrações a jusante do depósito, não se pode afirmar que este está controlado e estabilizado, uma vez que se verificam processos redox e alterações na qualidade da água amostrada especialmente após 
eventos chuvosos, possivelmente decorrente da lixiviação de compostos existentes no depósito. Isto merece atenção uma vez que existe a possibilidade de outros compostos normalmente não amostrados em campanhas de monito- ramento - como os contaminantes emergentes, amplamente encontrados em aterros (Masoner et al., 2014) - estarem sendo carreados para o entorno, proporcionando riscos à saúde pública e aos ecossistemas.

\section{AGRADECIMENTOS}

\section{À CAPES e à FAPESP pelo apoio financeiro no desenvolvimento desta pesquisa.}

\section{REFERÊNCIAS}

ÁLVARES, C.M.B. Contribuição ao conhecimento do meio físico da região do lixão de São Carlos-SP, através de estudos geológicos, geofísicos, topográficos e químicos. São Carlos, 2000. Dissertação (Mestrado) - Escola de Engenharia de São Carlos, Universidade de São Paulo.

APHA. Standard methods for the examination of water and wastewater. 22nd ed. Washington: American Public Health Association; AWWA, WEF., 2012.

BELEVI, H. \& BACCINI, P. Long-term behavior of municipal solid waste landfills. Waste Management and research, v. 7 , p. 43-56, 1989.

BJERG, P.L.; ALBRECHTSEN, H.J.; KJELDSEN, P., CHRISTENSEN, T.H.; COZZARELLI, I. The Groundwater Geochemistry of Waste Disposal Facilities. In: HOLLAND, H.D.; TUREKIAN, K.K. Environmental Geochemistry: Treatise on Geochemistry. 2 ed.. Elsevier Science, 2013.

BOSSOLAN, N.R.S. Aspectos ecológicos das populações bacterianas em águas subterrâneas sob o efeito de um aterro controlado. São Carlos, 1993. Dissertação (Mestrado), Universidade federal de São Carlos.

CETESB - Qualidade da água subterrânea no estado de São Paulo. São Paulo, CETESB, 2016. Disp. em: https://cetesb.sp.gov.br/aguas-subterraneas/wp-

content/uploads/sites/13/2013/11/Cetesb_QualidadeAguasSub terraneas2015_Web_20-07.pdf

CETESB - Relação de áreas contaminadas. Disp. em http://areas.contaminadas.cetesb.sp.gov.br

CHAKRABORTY, S., \& KUMAR, R.N. Assessment of groundwater quality at a MSW landfill site using standard and AHP based water quality index: a case study from Ranchi, Jharkhand, India. Environmental Monitoring and Assessment, v. 188, n. 6, 2016.

CHRISTENSEN, T.H. Attenuation of Leachate pollutants in groundwater. In CHRISTENSEN, T.H.; COSSU, R.; STEGMANN, R. Landfilling of waste: Leachate. London: Chapman and Hall, 1997.

CHRISTENSEN, T.H.; BJERG, P.L.; KJELDSEN, P. Natural Attenuation: A Feasible Approach to Remediation of Ground Water Pollution at Landfills?. Groundwater monitoring and remediation, 2000.

CHRISTENSEN, T.H.; KJELDSEN, P.; JENSEN, D. BJERG, P.L. Biochemistry of landfill leachate plumes: review. Applied Geochemistry, v. 16, n. 7-8, p. 659 -718, 2001.

COOK, P.G. \& HERCZEG, A.L. Environmental tracers in subsurface hydrology. New York, Springer Science Business Media, 2000.

ELIS, V.E. \& ZUQUETTE, L.V. Caracterização geofísica de áreas utilizadas para disposição de resíduos sólidos urbano. Revista Brasileira de Geociências, v. 32, n. 1, p. 119-134, 2002.

FAGUNDES, J.R.T. \& ZUQUETTE, L.V. Sorption behavior of the sandy residual unconsolidated materials from the sandstones of the Botucatu Formation, the main aquifer of Brazil. Environ Earth Sci., v. 62, p. 831-845, 2011.

FETTER, C.W. Contaminant Hydrogeology. Waveland Press, inc, 2018

FREITAS, A.L.S. Caracterização do aquífero Botucatu na região do Lixão de São Carlos-SP. São Carlos, 1996.
Dissertação (Mestrado) - Escola de Engenharia de São CarlosUniversidade de São Paulo,

GADOTTI, R.F. Avaliação da contaminação das águas superficiais e subterrâneas adjacentes ao lixão da cidade de São Carlos. São Carlos. 1997. 150p. Dissertação (Mestrado), Escola de Engenharia de São Carlos - Universidade de São Paulo,

GILBERTO, T.J. Estudos de Voçorocas aterradas por resíduos. Ribeirão Preto, 2007. Dissertação (Mestrado) Universidade de Ribeirão Preto.

GONÇALVES, A.R.L. Geologia ambiental da área de São Carlos. São Carlos, 1986. Tese (Doutorado), Escola de Engenharia de São Carlos - Universidade de São Paulo,

MASONER, J.R.; KOLPIN, D.W.; FURLONG, E.T.; COZZARELLI, I.M.; GRAY, J.L.; SCHWAB, E.A. Contaminants of emerging concern in fresh leachate from landfills in the conterminous United States. Environmental Science: Processes \& Impacts, v. 16, n. 10, p. 2335-2354, 2014.

MATSUZAKI, S.S. Aplicação de modelo computacional de escoamento de água subterrânea no lixão de São Carlos. São Carlos. 1998. 113 p. Dissertação (Mestrado) Escola de Engenharia de São Carlos -Universidade de São Paulo.

MENEZES, D.B. Diagnóstico dos impactos do lixão de São Carlos (SP) no meio físico. São Carlos, 1995. Dissertação (Mestrado), Escola de Engenharia de São Carlos - Universidade de São Paulo.

KJELDSEN, P.; BARLAZ, M.A.; ROOKER, A.P.; BAUN, A.; LEDIN, A.; CHRISTENSEN, T.H. Present and Long-Term Composition of MSW Landfill Leachate: A Review. Critical Reviews in Environmental Science and Technology, v. 32, n. 4, p. 297-336, 2002.

NEMATOLLAHI, M.J.; CLARK, M.J.R.; EBRAHIMI, P.; EBRAHIMI, M. Preliminary assessment of groundwater hydrogeochemistry within Gilan, a northern province of Iran. Environmental Monitoring and Assessment, v. 190, p. 242, 2018.

PASCHOALATO, C.F.P.R.; MARTINEZ, M.S.; FERREIRA, J.F.; BOCALON, E.C. Otimização de parâmetros analíticos a serem empregados no monitoramento de águas subterrâneas em aterros sanitários. In: CONGRESSO BRASILEIRO DE ENGENHARIA SANITÁRIA E AMBIENTAL, 21, 2001, João Pessoa. Atas...João Pessoa: Associação Brasileira de Engenharia Sanitária e Ambiental, 2001.

PELINSON, N.S. Avaliação de contaminantes em um lixão abandonado e seu entorno sob condições climáticas subtropicais. São Carlos, 2018. Tese (Doutorado), Escola de Engenharia de São Carlos - Universidade de São Paulo.

PMSC. Prefeitura Municipal de São Carlos. Investigação ambiental detalhada. (Relatório final) Coordenadoria Municipal de Meio Ambiente. São Carlos, 2011, 390 p.

PRZYDATEK, G. \& KANOWNIK, W. Impact of small municipal solid waste landfill on groundwater quality. Environmental Monitoring and Assessment, v. 191, p. 169 2019.

RANA, R.; GANGULY, R.; GUPTA, A.K. Indexing method for assessment of pollution potential of leachate from nonengineered landfill sites and its effect on ground water quality. 
Environmental Monitoring and Assessment, v. 190, n. 1, 2017.

RIOS, L. Estudo limnológico e fatores ecológicos em ribeirões e córregos da bacia do Ribeirão do Feijão (Estado de SP). São Carlos, 1993. Dissertação (Mestrado), Escola de Engenharia de São Carlos - Universidade de São Paulo.

SHINZATO, M.P.B. Mobilização de poluentes no maciço de resíduos de lixão desativado. São Carlos, 2014. 149 p. Tese (Doutorado), Escola de Engenharia de São Carlos Universidade de São Paulo.

SILVA, R.B.G. Estudo hidroquímico e isotópico das águas subterrâneas do aqüífero Botucatu no Estado de São Paulo. São Paulo, 1983. Tese (Doutorado), Instituto de Geociências Universidade de São Paulo.

SINDICO, F.; HIRATA, R.; MANGANELLI, A. The Guarani Aquifer System: From a Beacon of hope to a question mark in the governance of transboundary aquifers. Journal of Hydrology: Regional Studies, v. 20, p. 49-59, 2018
VAN BREUKELEN, B.M. \& GRIFFIOEN, J. Biogeochemical processes at the fringe of a landfill leachate pollution plume: potential for dissolved organic carbon, $\mathrm{Fe}(\mathrm{II}), \mathrm{Mn}(\mathrm{II}), \mathrm{NH} 4$, and CH4 oxidation. Journal of Contaminant Hydrology, v. 73, n. 1-4, p. 181-205, 2004.

VELOZO, R. Caracterização geológico-geotécnica do lixão desativado de São Carlos - SP, com auxílio da geofísica. São Carlos, 2006. Dissertação (Mestrado), Escola de Engenharia de São Carlos - Universidade de São Paulo, São Carlos.

Submetido em 16 de novembro de 2018 Aceito em 3 de maio de 2019 